I received duloxetine (60 mg once daily for 6 months) plus 50,000 unit oral cholecalciferol weekly for 8 weeks then monthly for 16 weeks. Group II received duloxetine ( $60 \mathrm{mg}$ once daily for 6 months) plus placebo. The patients were assessed at baseline and after 6 months of treatment by measuring serum levels of 25(OH)D, Fibromyalgia Impact Questionnaire (FIQ), Medical Outcomes Study Questionnaire Short Form 36 Health Survey (SF-36) \& Hospital Anxiety and Depression Scale (HADS). Results: Eighty six patients completed this study. There was no significant difference between all groups in demographic data, educational status and all baseline variants except serum levels of $25(\mathrm{OH}) \mathrm{D}$. After 6 months; there was significant improvement $(\mathrm{P}<0.05)$ in group I in serum levels of $25(\mathrm{OH}) \mathrm{D}$. There was significant improvement $(P<0.05)$ after 6 months in FIQ, SF-36 and HADS in both groups. There was significant better improvement $(\mathrm{P}<0.05)$ in group I than in group II in FIQ, SF-36 and HADS. The results of the study are summarized in table 1.

Table 1. Pre- and post-treatment assessment measures of the patient groups

\begin{tabular}{|c|c|c|c|c|}
\hline \multirow[t]{2}{*}{ assessment measures } & Baseline & Baseline & After 6 months & After 6 months \\
\hline & Group I & Group II & Group I & Group II \\
\hline 25(OH)D & $25.3 \pm 4.9 \mathrm{ng} / \mathrm{ml}$ & $26.8+5.3 \mathrm{ng} / \mathrm{ml}$ & $36.8+3.9 \mathrm{ng} / \mathrm{ml}$ & $25.6 \pm 3.4 \mathrm{ng} / \mathrm{ml}$ \\
\hline FIQ & $47.5 \pm 5.4$ & $46.7 \pm 6.7$ & $27.5 \pm 6.1$ & $38.5 \pm 7.3$ \\
\hline SF-36 (Total score) & $47.6 \pm 10.4$ & $47.0 \pm 9.9$ & $61.0 \pm 5.8$ & $54.8 \pm 5.3$ \\
\hline HAD anxiety & $8.2 \pm 0.6$ & $8.4 \pm 0.3$ & $7.1 \pm 0.7$ & $7.5 \pm 1.4$ \\
\hline HADS depression & $8.6 \pm 0.3$ & $8.6 \pm 0.9$ & $7.3 \pm 0.8$ & $7.7 \pm 1.4$ \\
\hline
\end{tabular}

Conclusion: Vitamin D supplement is effective as an adjuvant therapy in improving functional status, quality of life and psychological status in fibromyalgia patients with vitamin D insufficiency.

References:

[1] Abd Elghany S E et al, Regenerative injection therapy and repetitive transcranial magnetic stimulation in primary fibromyalgia treatment: A comparative study. Journal of Back and Musculoskeletal Rehabilitation -1 (2018) 1-8

[2] Maria Helde-Frankling, Linda Björkhem-Bergman. Vitamin D in Pain Management. Int. J. Mol. Sci. 2017, 18, 2170

Disclosure of Interests: : None declared

DOI: 10.1136/annrheumdis-2020-eular.487

\section{THU0472 CATHEPSIN S GENE EXPRESSION MEASURED IN THE PERIPHERAL BLOOD OF OSTEOARTHRITIC PATIENTS PRIOR TO SURGERY AS A BIOMARKER OF POST- OPERATIVE PAIN DEVELOPMENT}

E. Tchetina ${ }^{1}$, K. Glemba ${ }^{2}$, G. Markova ${ }^{1}$, E. Taskina ${ }^{3}$, M. Makarov ${ }^{2} .{ }^{1}$ Nasonova Research Institute of Rheumatology, Immunology and Molecular Biology, Moscow, Russian Federation; ${ }^{2}$ Nasonova Research Institute of Rheumatology, Orthopedics, Moscow, Russian Federation; ${ }^{3}$ Nasonova Research Institute of Rheumatology, Osteoarthritis, Moscow, Russian Federation

Background: Osteoarthritis $(O A)$ is a chronic rheumatic disease, which involves pain, limited inflammation, and local destruction of the knee joint. OA pain is a major clinical symptom, which limits working capacity and denotes an important indication for joint replacement in the end-stage OA. In spite of significant number of positive outcomes, chronic postoperative pain represents a major adverse consequence of surgery, which is observed in $10-40 \%$ of OA patients. Therefore, identification of patients potentially capable of developing chronic postoperative pain prior to surgery could significantly improve therapy outcome. Recently we hypothesized that genes related to pain sensitization whose expression is upregulated in about $10-40 \%$ of the examined end-stage OA patient cohort might be responsible for postoperative pain. Retrospective analysis of gene expression in the peripheral blood of end-stage OA patients before joint replacement surgery revealed that expression of cathepsins S and K, caspase 3, and MMP-9 genes might be associated with postoperative pain development [Ann Rheum Dis,78, suppl 2:A520]

Objectives: To examine the validity of our hypothesis in the prospective study. Methods: We examined peripheral blood of 26 healthy volunteers (average age $55 \pm 8.3$ years old) and 40 end-stage OA patients (average age $56.5 \pm 8.9$ years old) undergoing joint replacement surgery. Patients were examined before and 6 months after surgery. Pain was assessed prior to surgery using VAS index and neuropathic pain questionnaires DN4 and PainDETECT. Functional activity was evaluated by WOMAC. After surgery pain indices according to VAS of $30 \%$ and higher were considered. MMP-9 and caspase 3 protein levels were quantified by ELISA. Total RNA isolated from whole blood was used in expression studies for caspase 3; metalloproteinase (MMP)-9; cathepsins $\mathrm{K}$ and $\mathrm{S}$ genes. These were performed with quantitative real-time RT-PCR.
Results: Out of 40 patients pain complaints were obtained from 9 patients $(22,5 \%)$ after 6 months' post-surgery. Prior to surgery all the examined genes were significantly upregulated in the patients who developed post-operative pain compared to healthy controls and those subjects who did not develop pain after surgery. However, no difference in the levels of the examined pain-related and functional indices in patients, who developed pain or not, was noted before surgery. ROC curve analyses confirmed significant associations $(p<0.05)$ between expressions of the examined genes prior to surgery with the likelihood of pain development after surgery. The cut-off values for the examined gene expressions were 11.34 for cathepsin S (sensitivity of 0.89 and specificity of 0.76 ), 10.11 for caspase 3 (sensitivity of 0.86 and specificity of 0.65 ), 10.09 for cathepsin $\mathrm{K}$ (sensitivity of 0.86 and specificity of 0.78 ). Moreover, among the examined genes cathepsin $\mathrm{S}$ expression was the most informative predictor of postoperative pain development $[\mathrm{AUC}=0.857,95 \% \mathrm{Cl}(0.708-1.000)]$.

Conclusion: High cathepsin $\mathrm{S}$ gene expression in the peripheral blood of the end-stage OA patients measured prior to joint replacement surgery could serve an important biomarker of post-operative pain development.

Disclosure of Interests: : None declared

DOI: 10.1136/annrheumdis-2020-eular.2187

\section{THU0473 IS PAIN INTENSITY ASSOCIATED WITH EARLY MORTALITY IN PATIENTS WITH PSORIATIC ARTHRITIS?}

J. Vela $^{1,2}$, R. Cordtz ${ }^{1}$, S. Kristensen ${ }^{1,3}$, K. Kjær Petersen ${ }^{4,5}$, L. Arent-Nielsen ${ }^{4}$, C. T. Torp-Pedersen ${ }^{6}$, L. Dreyer ${ }^{1,3,7} .{ }^{1}$ Aalborg University Hospital, Aalborg, Denmark; ${ }^{2}$ Clinical Institute, Aalborg University, Aalborg, Denmark; ${ }^{3}$ Clinical institute, Aalborg University, Aalborg, Denmark; ${ }^{4}$ Center for Sensory-Motor Interaction, Aalborg University, Aalborg, Denmark; ${ }^{5}$ Center for Neuroplasticity and Pain, Aalborg University, 9000, Denmark; ${ }^{6}$ Northzealands Hospital Hillerød, Department of Cardiology, Hillerød, Denmark; ${ }^{7}$ DANBIO Registry, Glostrup, Denmark

Background: Studies regarding excess mortality among patients with psoriatic arthritis (PsA) are conflicting due to the heterogenous nature of the disease. Thus, identifying risk factors for mortality is crucial, but few studies have examined these in PsA. Presence of chronic pain can cause excess mortality and since pain is prevalent among patients with PsA this association should be explored.

Objectives: To investigate whether higher cumulative pain intensity is associated with an excess mortality ratio in patients with PsA.

Methods: A nested case-control study was performed using data from the national Danish healthcare registers and the DANBIO rheumatology regis ter. Cases were patients who died while followed in routine care. Cases were matched on sex, year of birth and calendar period of DANBIO entry with up to five controls. The main exposure of interest was the mean pain intensity (all causes) reported during the time followed in routine rheumatology practice. The pain intensity was measured on a visual analogue scale (VAS) ranging from 0 (no pain) to 100 (worst imaginable pain). Conditional logistic regression was used to calculate the odds of mortality per 5 unit increase in VAS pain while adjusting for inflammatory markers.

Results: The Danbio PsA cohort consisted of 8019 patients. In total, 266 cases i.e. PsA patients who died during the observational period, were identified and matched with 1198 controls ( 4.5 controls per case). Increasing pain intensity was associated with increased odds of mortality (OR 1.05, 95\% Cl 1.01 to 1.09) in the crude model, but the association disappeared when adjusting for age, sex, calendar time, socioeconomic status, average c-reactive protein and swollen joint count during the observation period (OR $0.98,95 \% \mathrm{Cl} 0.93-1.03$ ).

Age, average CRP, biological DMARD use, glucocorticoid use, and comorbidities (see table) increased the odds of mortality.

Table regression estimates from fully adjusted model

\begin{tabular}{lcc}
\hline & Odds Ratio & \\
\hline Age & 2,73 & $95 \% \mathrm{Cl}$ \\
C-reactive protein & 1,05 & $1,60-4,68$ \\
Swollen joint count & 1,08 & $1,03-1,07$ \\
Health assessment questionnaire & 1,25 & $0,97-1,22$ \\
bDMARD use & 2,62 & $0,84-1,86$ \\
cDMARD use & 0,69 & $1,51-4,57$ \\
Glucocorticoid use & 3,90 & $0,46-1,03$ \\
Chronic obstructive pulmonary disease & 2,19 & $2,51-6,05$ \\
Diabetes mellitus & 2,65 & $1,20-4,02$ \\
Cancer & 6,15 & $1,62-4,31$ \\
Cardiovascular disease & 2,61 & $3,88-9,76$ \\
& & $1,71-3,97$
\end{tabular}

Conclusion: These results indicate that experienced pain in itself is not associated with excess mortality. Age, recent glucocorticoid use, biological DMARD 